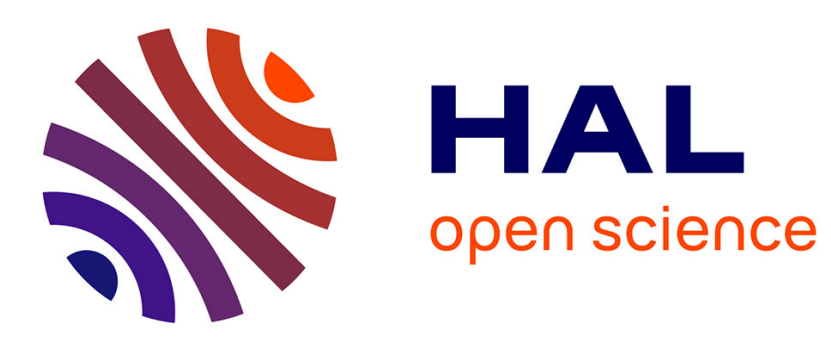

\title{
Simulation of cylindrical electron cyclotron wave resonance argon discharges
}

S Sfikas, E Amanatides, D Mataras, D Rapakoulias

\section{To cite this version:}

S Sfikas, E Amanatides, D Mataras, D Rapakoulias. Simulation of cylindrical electron cyclotron wave resonance argon discharges. Journal of Physics D: Applied Physics, 2011, 44 (16), pp.165204. 10.1088/0022-3727/44/16/165204 . hal-00613270

\section{HAL Id: hal-00613270 \\ https://hal.science/hal-00613270}

Submitted on 4 Aug 2011

HAL is a multi-disciplinary open access archive for the deposit and dissemination of scientific research documents, whether they are published or not. The documents may come from teaching and research institutions in France or abroad, or from public or private research centers.
L'archive ouverte pluridisciplinaire HAL, est destinée au dépôt et à la diffusion de documents scientifiques de niveau recherche, publiés ou non, émanant des établissements d'enseignement et de recherche français ou étrangers, des laboratoires publics ou privés. 


\title{
Simulation of Cylindrical Electron Cyclotron Wave Resonance Argon Discharges
}

\author{
S. Sfikas, E. Amanatides, D. Mataras and D. Rapakoulias \\ Plasma Technology Laboratory, Department of Chemical Engineering, \\ University of Patras, P.O. Box 1497, 26504, Patras Greece \\ E-mail: $\underline{\text { dim@plasmatech.gr }}$
}

\begin{abstract}
A fluid model of a cylindrical electron cyclotron wave resonance (ECWR) Argon discharge is presented. The results for a 1 mTorr Argon discharge were checked against the analytical theory, simulation and experimental data. The basic plasma properties as power dissipation, magnetic field, electric potential, electron density and temperature were very well reproduced by using pre-defined boundary conditions for the magnetic potential. The results of this model were further used as inputs for the simulation of plasma expansion into a diffusion region, allowing thus fast and complete modeling of a typical ECWR plasma reactor.
\end{abstract}




\section{Introduction}

Electron Cyclotron Wave Resonance (ECWR) plasma sources have recently found several applications in materials processing [1]. Plasma Oxidation and Plasma Enhanced Chemical Vapor Deposition (PECVD) of a-C:H, c-BN, nc-Si:H thin films are a few examples of the processes where ECWR has already been applied successfully [2-6]. The main advantage of these types of sources is that radio-frequency coupling into the inductively coupled plasma is enhanced in this case by the ECWR effect, leading to higher plasma densities compared to capacitive or purely inductive discharges [1].

The ECWR effect in plasma reactors is induced by the superposition of a small static magnetic field perpendicular to the axis of the radio-frequency excited coil and in parallel to the inductively excited electron cyclotron waves [7]. ECWR utilizes the propagation branch for generator frequencies $\left(\omega_{\mathrm{rf}}\right)$ well below the cyclotron frequency $\left(\omega_{\mathrm{ce}}\right)$ in the dispersion diagram of the "whistler" or right-handed mode; thus it has to be well distinguished from the Electron Cyclotron Resonance (ECR) effect. The superposition of a small static magnetic field produces a slight anisotropy on the plasma electrical properties. Resonant plasma excitation by electron cyclotron waves is possible, when an odd number of half of the wavelength of the propagating cyclotron wave fits the plasma thickness in the direction of the wave vector (i.e. the formation of a standing wave pattern) $[1,7,8]$. The latter ensures a more efficient plasma heating and higher plasma densities compared to conventional capacitive and inductive sources.

In an effort to understand and explain the resonance effect, the rather complicated anisotropic electrodynamics of these systems were studied through various approaches. Thus, theoretical studies [9], analytical solutions $[8,10]$ and simulations [11] together with experimental findings $[12,13]$ have been used to establish the theory behind the ECWR effect. However, self-consistent modeling of such complex gas discharges is still a challenging task. It is very important for further consideration of these complex plasma sources in industrial applications[14]. 
We extend our recently reported fluid model of an ECWR discharge in a 3d infinite slab [15] to more realistic cylindrical plasma reactor geometries including also the plasma expansion region, which is very important for the simulation of discharges used in materials processing. Model results for $1 \mathrm{mTorr}$ Ar discharges are presented and compared to other theoretical and experimental findings.

\section{Model Description}

In figure 1 the reactor geometry is presented, including the bounding dielectric walls, together with the static $\left(\mathrm{B}_{\mathrm{st}}\right)$ and high-frequency $(h f)$ alternating magnetic field $\left(\mathrm{H}_{\mathrm{z}}\right)$ directed along the $\mathrm{x}$ and z-axis, respectively. The geometry of the chamber, a cylindrical glass vessel of diameter $\mathrm{d}_{\text {cyl }}=0.095 \mathrm{~m}$ and height $\mathrm{h}=0.095 \mathrm{~m}$ filled with $1 \mathrm{mTorr}$ Argon is similar to those in Ref's [10-13]. A single sector coil carrying a current of frequency $\omega_{\mathrm{rf}}=2 \cdot \pi \cdot 27.12 \mathrm{MHz}$ is positioned immediately outside the bounding walls. We introduced a Cartesian coordinate system with the $\mathrm{x}$-axis in the direction of the stationary external field $\mathrm{B}_{\mathrm{st}}$ and the $\mathrm{z}$-axis coinciding with the axis of the infinitely long cylinder.

In order to reproduce the electromagnetic field in this cylindrical configuration, we have adopted the estimation for the right-hand polarized (RHP) wave propagating along the field $B_{\text {st }}$ as in Ref. [9]:

$$
\mathrm{B}_{\mathrm{RHP}}=\mathrm{B}_{\mathrm{RHP} 0} \cdot \cos \left(\mathrm{K}_{\mathrm{RHP}} \cdot \mathrm{x}\right) \cdot \cos \left(\pi \cdot \mathrm{y} / \mathrm{d}_{\mathrm{cyl}}\right)
$$

where, $\mathrm{K}_{\mathrm{RHP}}$ and $\mathrm{B}_{\mathrm{RHP} 0}$ are the wavenumber and the peak value of the RHP wave. The value of $\mathrm{B}_{\mathrm{st}}$ in resonance can be calculated by the formula proposed in Ref. [10].

$$
N_{\text {res }}=\frac{\pi^{2} \cdot c^{2} \cdot m_{e} \cdot \varepsilon_{0}}{e^{2} \cdot} \cdot \frac{p^{2}\left(\lambda_{i}\right) \cdot t^{*} \cdot\left(\beta_{r e s}-t^{*}\right)^{3} \cdot t_{\|}^{-2}}{\left(\beta_{r e s}-t^{*}\right)^{2}+p^{2}\left(\lambda_{i}\right) \cdot t^{*} \cdot\left(\pi / t_{\|}\right)^{2} \cdot\left(k T_{e} / m_{e} \cdot \omega^{2}\right)} .
$$

where, $\beta_{\text {res }}$ the electron cyclotron frequency $\omega_{c}$ normalized to the angular frequency

of the generator $\omega, c$ is the velocity of light, $m_{e}$ the electron mass, $\varepsilon_{0}$ the dielectric 
constant in vacuum, $p^{2}\left(\lambda_{i}\right)$ a correction term for the nonhomogeneous density

distribution, $t^{*}$ a correction term accounting for the rectangular cross section of the plasma volume and $T_{e}$ the electron temperature. In order to approximate the circular cross section of a cylindrical discharge by a square, Szuszczewicz proposed a scheme that involves simple averaging of the values of plasma dimension $t \|$ parallel to the static magnetic field over the circumference of the discharge. His resulting adaptation was the approximation of the circular cross section of diameter $\cdot d_{c y l}$ by a square of side $t \|=2 \cdot d_{c y l} / \pi$.

Implementing this approximation, we set $t^{\star}=1.41$ for the correction term, $t \perp=t \|=2 \cdot d_{c y l} / \pi$ for the plasma dimension perpendicular $(t \perp)$ and parallel $(t \|)$ to the static magnetic field, $\mathrm{p}^{2}\left(\lambda_{\mathrm{i}}\right)=2.09$ for the correction term for a non-homogeneous density and $\mathrm{T}_{\mathrm{e}}=80000{ }^{\circ} \mathrm{K}$ for the electron temperature. Setting the value of the desired electron density at resonance $\left(\mathrm{N}_{\mathrm{res}}\right)$ to $1 \cdot 10^{17} \mathrm{~m}^{-3}$, eq. $(1 \mathrm{~b})$ results to $\beta_{\text {res }}\left(=\frac{\omega_{\mathrm{c}}}{\omega_{\mathrm{rf}}}=\frac{\mathrm{e} \cdot \mathrm{B}_{\mathrm{st}}}{\mathrm{m}_{\mathrm{e}} \cdot \omega_{\mathrm{rf}}}\right)=2.136$, which corresponds to a value of 2.07 mTesla (20.7 Gauss) for the stationary magnetic field. Moreover, for $\mathrm{K}_{\mathrm{RHP}=} 51.88 \mathrm{~m}^{-1}$, half a wavelength of the RHP wave fits into a slab of width $d_{\text {slab }}=2 \cdot d_{c y l} / \pi$. The peak value ( $\left.\mathrm{B}_{\mathrm{RHP} 0}\right)$ of the RHP wave, was calculated to be nearly $64.16 \%$ of the value of the alternating magnetic field $B_{R F}$ at the walls, using back-substitution of known values $\left(\mathrm{K}_{\mathrm{RHP}}\right.$ and $d_{\text {cyl }}$ ) in eq. (9a) of ref [8]. In addition, the value of the alternating magnetic field $\mathrm{B}_{\mathrm{RF}}$ at the walls was set to 0.32 mTesla (3.2 Gauss), resulting from a of 24.2 Ampere (peak value) coil current.

For the magnetic vector potential, we have used a specific set of boundary conditions, under the following assumptions:

- The RHP component of the wave is responsible for electron heating.

- The z-component of the alternating magnetic field, induces a cross sectional electric field (i.e. field components in the xy-plane) which is of major importance compared to the axially induced field. 
- Plasma conductivity in the direction of the stationary field $\left(\sigma_{x x}\right)$ is predominant: Inductive power absorption occurs mainly in regions where the $\mathrm{x}$-component of the induced electric field prevails.

- Modification of the effective collision frequency introduced in [15] can lead to the substitution of plasma tensor conductivity by a scalar.

The $A_{x}$ and $A_{y}$ components of the magnetic potential can be expressed as:

$$
\begin{aligned}
& A_{x}=-c_{1} \cdot c_{2} \cdot\left|\sin \left(b_{x} \cdot K_{R H P} \cdot r \cdot c_{3}^{2}\right)\right| \cdot A_{\text {max }} \cdot(y / r) \\
& A_{y}=c_{1} \cdot c_{2} \cdot\left|\sin \left(b_{y} \cdot K_{R H P} \cdot r\right)\right| \cdot A_{\text {max }} \cdot(x / r)
\end{aligned}
$$

where the $e^{j \omega t}$ time dependence has been eliminated and $\mathrm{A}_{\max }$ is the maximum value of the magnetic potential calculated to be $2.097 \cdot 10^{-6} \mathrm{Weber} / \mathrm{m}$ [15]. The following values have been used for the dimensionless numerical constants $b_{x}, b_{y}, c_{1}, c_{2}, c_{3}$ in order to reproduce the magnetic field of Eq. (1a): $c_{1}=1.9, c_{2}=0.58, c_{3}=(2 / \pi), b_{x}=1.357203, b_{y}=1.2748$. The cylinder radius was also defined as $\mathrm{r}=d_{c y l} / 2$.

The magnetic field can be also approximately reconstructed using first-order Bessel functions for the magnetic potential:

$$
\begin{aligned}
& A_{x}=-c_{1} \cdot J_{1}\left(K_{R H P} \cdot r \cdot c_{3}\right) \cdot A_{\text {max }} \cdot(y / r) \\
& A_{y}=c_{1} \cdot J_{1}\left(K_{R H P} \cdot r / c_{3}\right) \cdot A_{\text {max }} \cdot(x / r)
\end{aligned}
$$

The spatial distribution of the high-frequency magnetic field $\mathrm{B}_{\mathrm{z}}$, calculated from the difference $\frac{\partial A_{y}}{\partial x}-\frac{\partial A_{x}}{\partial y}$ in Eqs. $(2,3)$ is presented in Fig. 2(a). The distribution has the form of the analytical expression given in Eq. (1a). The high-frequency magnetic field $\mathrm{B}_{z}$ can be reproduced close to the $\mathrm{x}$-axis using the analytical theory for $\mathrm{B}_{\mathrm{z}}$, while in the direction perpendicular to $\mathrm{B}_{s t}$ it is close to the values predicted for the imaginary $\left(\mathrm{B}_{\mathrm{y}}\right)$ component in ref. [8]. Moreover, close to the y-axis, the magnetic field resembles the simulation results of ref. [11] at the phase of vanishing coil current in the $r f$ cycle. Nevertheless, Krimke et al [11] have introduced a discharge model with predefined plasma properties $\left(\mathrm{N}_{\mathrm{e}}, v_{\mathrm{e}-\mathrm{n}}, \vec{\varepsilon}_{p}, \vec{\sigma}_{p}\right)$, within a 
static magnetic field $\left(\mathrm{B}_{\mathrm{st}}\right)$ of $2.55 \mathrm{mTesla}$, corresponding to $\beta_{\mathrm{res}}=2.63$ which is quite different from the experimental value.

The modulus of the cross sectional induced electric field calculated as $E_{R F}=\sqrt{E_{x}^{2}+E_{y}^{2}}=\omega_{R F} \cdot \sqrt{A_{x}^{2}+A_{y}^{2}}$ is shown in the map of Fig. 2(b). The coil surface charge irrotational field [11] will be shielded by the sheath and was not taken into account in the simulation since it is not contributing to electron heating. Two fields of dipole nature ( $E_{x}$ and $E_{y}$ components) are superposed in $E_{R F}$ so the quadrupolar character of the field that was revealed in [11] is still present-yet distorted.

The inductive power dissipation is calculated as:

$$
P_{R F}=\frac{1}{2} \cdot \operatorname{Re}\left(\sigma_{p}\right) \cdot E_{R F}^{2}
$$

Inductive power dissipation mainly occurs in regions of high plasma conductivity and field strength. Calculation of the components of conductivity tensor using the Appleton-Hartree theory [16] leads to $\sigma_{x x} / \sigma_{y y} \cong 2.235$, for $\mathrm{N}_{\mathrm{res}}=1 \cdot 10^{17} \mathrm{~m}^{3}, \omega_{\mathrm{c}}=363.95 \mathrm{MHz}, \omega_{\mathrm{rf}}=2 \cdot \pi 27.12 \mathrm{MHz}$, $\omega_{\mathrm{p}}=17.8 \mathrm{GHz}$ and the collision frequency as calculated in [17] to be $v=4 \cdot 10^{6} \mathrm{~s}^{-1}$ ). The region of interest lies in the $y$-axis which is perpendicular to the static field $\mathrm{B}_{\mathrm{st}}$. In order to incorporate this power absorption non-uniformity, we have inserted a function $\mathrm{g}(\mathrm{r})=\left(\mathrm{dE}_{\mathrm{x}} / \mathrm{d}_{\mathrm{r}}\right) / \mathrm{E}_{\mathrm{x} 0}$ in eq. (4) of Ref. [15] for the effective collision frequency $v_{\text {eff: }}$ :

$$
v_{e f f}=\left[1+\frac{1}{g(r)}\left(\frac{\omega_{c} \cdot \frac{N_{e}}{N_{e R E S}}}{v}\right)^{2}\right] \cdot v
$$

where, $g(r)$ is the normalized gradient of the $\mathrm{x}$-component of the induced electric field $\mathrm{E}_{\mathrm{x}}$, and it essentially represents the influence of the $\mathrm{E}_{\mathrm{x}}$ field in electron mobility: The $\mathrm{x}$-component of the electric field tends to affect the electron motion in a direction parallel to the $\mathrm{B}_{\mathrm{st}}$ field; as a consequence, the electron mobility and plasma conductivity will be considerably increased. The penetration distance for the $\mathrm{E}_{\mathrm{x}}$ field was set to $\delta=0.017 \mathrm{~m}$, which corresponds to the 
normal skin depth value (non-locality parameter $\Lambda<<1$ [18]). Modifications in $v_{\text {eff }}$ are taking place only within this region of $E_{x}$ penetration. Moreover, the penetration depth for $E_{x}$ was assumed to vary in the interval $[0 ; 0.017]$ as $\delta=0.017|\mathrm{y} / \mathrm{r}|$, because of the decreasing influence of the $\mathrm{E}_{\mathrm{x}}$ component as $\mathrm{y} \rightarrow 0$.

For the case presented here, the domain was split into 1152 cells. The magnetic field was solved in the frequency domain; while for the electrostatic field and the charged species mass, momentum (for ions) and energy (for electrons) balance we have employed a time step of 1.8436 ns. Finally, for neutral species a time step of $184.36 \mathrm{~ns}$ was used. The system of equations for the particle, momentum and energy balances was obtained from moments of the Boltzmann transport equation and was coupled with Poisson (electrostatic field calculation) and Maxwell ( $h f$ electromagnetic field calculation) equations; finally, it was complemented by a set of boundary conditions concerning the species' densities as well as the electrostatic potential as in [15].

\section{Results and discussion}

In figure 3(a) we compare the results of our simulation for the electron density (spatial map with dark colored curves of constant density), with the experimental findings presented in fig. 7 of ref. [12] for $\mathrm{N}_{\mathrm{res}}=1 \cdot 10^{17} \mathrm{~m}^{-3}, \beta=2.0, \mathrm{z}=0$ : The variation of the ion current to the Langmuir probe between its mean and maximum value, is displayed by the superimposed light colored arcs for $r=1.0,2.0,3.0,4.0 \mathrm{~cm}$, representing higher electron density regions. The alignment of the regions of maximum electron density in the simulation is in good agreement with experiment in the main volume of the discharge and the net result of the anisotropy in charged particle diffusion coefficient is closely reproduced. In ECWR sources, the electron density profile is a decreasing function of the radius $r$ in the direction perpendicular to $\mathrm{B}_{\mathrm{st}}$ as resonance is approached, resulting in an increasing in-homogeneity of the extracted ion beam. This effect is demonstrated in figure 3(b): plasma density experimental data [12] collected in the center of the discharge along the diameter which is perpendicular to $\mathrm{B}_{\mathrm{st}}(\beta=0,1.5$, dotted lines) are compared to the simulation results $\left(\beta_{\text {res }}=2.13589\right.$, straight lines $)$. The normalized 
electron density radial profile $\left(N_{e}(x), \beta=2.13\right)$ along the static field is in fair agreement with the experimental data radial profile without the static field $\left(N_{e}(y), \beta=0\right)$ as it should due to the unaffected electron motion along the lines of force. The radial profile of the simulated electron density $\left(N_{e}(y), \beta=2.13\right)$ perpendicular to the static field is off the experimental values $\left(N_{e}(y), \beta=1.5\right)$; nevertheless, due to the hindered electron motion perpendicular to the static magnetic field, the simulated profile for $N_{e}(y)$ is sharper than for $N_{e}(x)$, as it should. The difference from the experimental findings can be attributed to the different plasma density symmetry properties at non-resonant values $\left(\beta_{\text {res }}>\beta=1.5\right)$ where the alignment of maximum density regions is along the static magnetic field [12].

The simulation results for the inductive power dissipation along $\left(\mathrm{P}_{\mathrm{RF}}(x)\right)$ and perpendicular $\left(\mathrm{P}_{\mathrm{RF}}(y)\right)$ to the static field are presented in fig. $4(\mathrm{a})$ : The radial profiles are entirely different due to the anisotropy of the conductivity in the plasma calculated as:

$$
\sigma_{p}=\frac{\varepsilon_{0} \cdot \omega_{p}}{v_{e f f}+i \cdot \omega}
$$

where $\omega_{p}$ is the electron plasma frequency.

The map of $\mathrm{P}_{\mathrm{RF}}$ distribution is shown in fig. 4(b). The regions of maximum power dissipation are aligned along the $y$-axis; the curved skin layer resulted in a power absorption decreasing as $x \rightarrow \pm r$ in a way comparable to the simulation results of Ref. [11]. A total power dissipation of $46 \mathrm{~W}$ was calculated from volume integration for the vessel of height $\mathrm{h}=0.095 \mathrm{~m}$, assuming homogeneity in the cylinder's axial direction. This value is almost $60 \%$ of the $80 \mathrm{~W}$ of total dissipated power referred in experimental [12] and simulation [11] results; however in an experimental arrangement [13] a low level $h f$ generator output power $P_{h f} \cong 50$ Watts was reported for the fundamental $(\beta=2.0)$ resonance.

Furthermore, the model prediction of electron temperature $T_{e}$ is presented in fig. 5(a). The simulated profiles demonstrate a deep $4 \mathrm{eV}$ minimum at the center of the discharge as a consequence of the hindered electron transport in the main volume. This value is close to experimental and simulation results reported in $[14,19]$ for a $\left(\mathrm{Ne} \approx 1^{\cdot} 10^{17} \mathrm{~m}^{-3}, \mathrm{p}=1 \mathrm{mTorr}\right.$, 
$\omega_{r f}=2 \pi 29 \mathrm{MHz}, \mathrm{B}_{\mathrm{st}}=20$ Gauss, $\mathrm{P}_{\mathrm{RF}}=100 \mathrm{~W}$ ) magnetized inductively coupled discharge configuration. The spatial anisotropy in energy transfer to electrons is reflected to the different maximum values and gradient of $\mathrm{T}_{\mathrm{e}}$ along $(x)$ and transverse $(y)$ to the static magnetic field $B_{s t}$. Although the power dissipation takes place in the direction perpendicular to the static magnetic field (fig. 4(b)), discharge heating seems to take place principally along the lines of force $\left(T_{e}(x)\right.$ curve) where electron motion is undisturbed. The minimum in electron temperature at the center of the discharge can also be attributed to low energy electrons trapped in the electrostatic potential $\Phi$ which exhibits spatial homogeneity and its' radial profiles are presented in fig. 5(b). It has a maximum value of $\Phi_{\max } \cong 36.5$ Volts and it can be approximately reproduced by eq. (9) for the ambipolar potential to account for a nonuniform plasma density:

$$
\Phi(x)=\Phi_{\max } \cdot\left\{1-\left\{\left[(2 \cdot x)+\mathrm{d}_{\mathrm{cyl}}\right] / \mathrm{d}_{\mathrm{cyl}}-1\right\}^{6}\right\}, \quad x \in\left[-\mathrm{d}_{\mathrm{cyl}} / 2, \mathrm{~d}_{\mathrm{cyl}} / 2\right]
$$

The coupling of the Poisson's relation to the fluid code was of great importance; otherwise the assumption of quasi-neutrality and ambipolarity led always to the underestimation of the electrostatic potential. The electrostatic potential distribution and range was calculated to be close to hybrid (fluid/kinetic) $[20,21]$ simulation results $\left(\mathrm{p}=1 \mathrm{mTorr}, \mathrm{Ne} \cong 10^{17} \mathrm{~m}^{-3}\right.$ ), of a nonmagnetized low pressure ICP discharge [22]. The spatial distribution and the range of the simulated electron temperature in fig. 5(a) are also comparable to the previous ref. [22].

Moreover, the model has been extended to include the plasma expansion into a diffusion region and towards the process chamber results for the 1 mTorr Ar ECWR plasma. The simulation geometry is similar with the one presented in ref. [23]. As an inlet of this diffusion regime, the bottom of the source (coil) was considered and the results of the previous analysis concerning the profiles along the $\mathrm{x}$-axis of electron and ion density, electron temperature and potential were used as boundary conditions. Perfectly conducting wall at a distance of $25 \mathrm{~cm}$ from the bottom of the coil was considered in order to achieve a faster convergence. The results for the spatial distribution of electron density and potential from the center of the source towards the reactor wall are shown in figures 6 (a) and (b), respectively. In the same 
figures, a comparison of the data with the experimental results and the fitting of the spherical plasma expansion model $[23,24]$ is included.

Even though the model nicely reproduces the rapid plasma degradation, electron density is overestimated especially for distances higher than $5 \mathrm{~cm}$ from the source (figure 6a) while a better agreement was found for the variation of potential (figure 6b) for distances higher than $10 \mathrm{~cm}$. Consideration of the chamber and source walls as dielectrics seems to give slightly better results but it also leads to much higher computational time.

Further work is in progress with modified inlet conditions and more complicated gas phase and surface chemistry in order to validate and apply this fast converging code in more complicated chemical systems.

\section{Conclusion}

A fluid model of low pressure ECWR Argon discharges, based on a modified effective collision frequency and predefined boundary conditions for the magnetic potential was developed. The model predictions are in good agreement with other experimental measurements and simulations for Ar discharges, allowing for rapid estimation of basic plasma properties such as electron density, temperature and plasma potential. The simulation outputs can be used as inputs in a plasma diffusion model which are in reasonable agreement with experimental data and a spherical plasma expansion model [10].

The development of this fluid model is constantly under the perspective of its extension in order to simulate complex chemical gas phase and surface processes as deposition of amorphous and microcrystalline silicon from mixtures of silane in hydrogen.

\section{REFERENCES}

[1] Oechsner H 2009 Vacuum 83727

[2] Ruijun Z and Hongtao M 2006 Mater. Sci. 411705

[3] Toth A, Mohai M, Ujvari T and Bertoti I 2005 Thin Solid Films 482183 
[4] Awakowicz P, Schwefel R, Scheuberta P and Benstetter G 2001 Surf. Coat. Technol. 142-

144342

[5] Lai DF, Robertson J and Milne WI 2001 Thin Solid Films 383220

[6] Scheib M, Schroder B and Oechsner H 1996 J. Non-Cryst. Solids 198-200 895

[7] Pfeiffer B 1966 J. Appl. Phys. 371628

[8] Pfeiffer B 1966 J. Appl. Phys. 371624

[9] O. Sager, PhD Thesis, Technische Hochschule Zurich, 1970.

[10] Szuszczewicz EP 1971 J. Appl. Phys. 424794

[11] Krimke R and Urbassek HM 1997 Plasma Sources Sci. T. 6415

[12] Szuszczewicz EP and Oechsner H 1972 Phys. Fluids 152240

[13] Oechsner H 1974 Plasma Physics 16835

[14] Polomarov O, Theodosiou C, Kaganovich I, Economou D and Ramamurthi B 2006 IEEE T. Plasma Sci. $\mathbf{3 4} 767$

[15] Sfikas S, Amanatides E, Mataras D ${ }^{*}$ and Rapakoulias D 2007 IEEE T. Plasma Sci. 35 1420

[16] Seshadri S, Fundamentals of Plasma Physics, New York: Elsevier, 1973.

[17] Chen F 2001 Phys. Plasmas 83008

[18] Lieberman M and Godyak V 1998 IEEE T. Plasma Sci. 26, 955.

[19] Godyak V and Alexandrovich B 2004 Phys. Plasmas 113553

[20] Kolobov V and Arslanbekov R 2003 Microelectron. Eng. 69606

[21] Kolobov V 2003 Comp. Mater. Sci. 28302

[22] Kolobov V and Arslanbekov R Simulation of Electron Kinetics in Gas Discharges", 2005 Workshop on Nonlocal, Collisionless Electron Transport in Plasmas, Presentation Notes, Princeton, NJ, August 2005.

[23] Vartolomei V Hannemann M Steffen H and Hippler R Intern. Conf. on Phenomena in Ionized Gases (2003)

[24] Hippler R Kredl and Vartolomei V 2009 Vacuum 83732 


\section{FIGURE CAPTIONS}

Figure 1: Geometrical model of the axially infinite plasma cylinder, with the axial component of the simulated alternating magnetic field $H_{z}$ and the superimposed static magnetic field $B_{s t}$.

Figure 2: Maps of (a) the simulated alternating magnetic field $B_{\mathrm{Z}}$ (normalized to the field value at the walls) and (b) the magnitude of the induced electric field, resulting from the specific boundary conditions used for the magnetic potential.

Figure 3: (a) Map of electron density $N_{\mathrm{e}}$ as calculated and compared to the experimental curves [12] of higher density regions (light colored lines) at radius $r=1$, 2, 3 and $4 \mathrm{~cm}$ and (b) Electron density profiles along $(x)$ and transverse $(y)$ direction to the static magnetic field $B_{s t}$ as calculated in the present simulation and experimentally measured in [12].

Figure 4: (a) Inductive power dissipation PRF profile, along (x) and transverse (y) to the static magnetic field Bst.(b) Inductive power dissipation PRF spatial map.

Figure 5: (a) Electron temperature $T_{\mathrm{e}}$ and (b) electric potential $\Phi$ profiles, along $(x)$ and transverse $(y)$ to the static magnetic field $B_{s t}$.

Figure 6: (a) Electron density and (b) plasma potential as a function of the distance from the center of the coil towards as calculated in the present simulation and compared to the spherical expansion model and experimental measurements of $[23,24]$. 


\section{FIGURES}

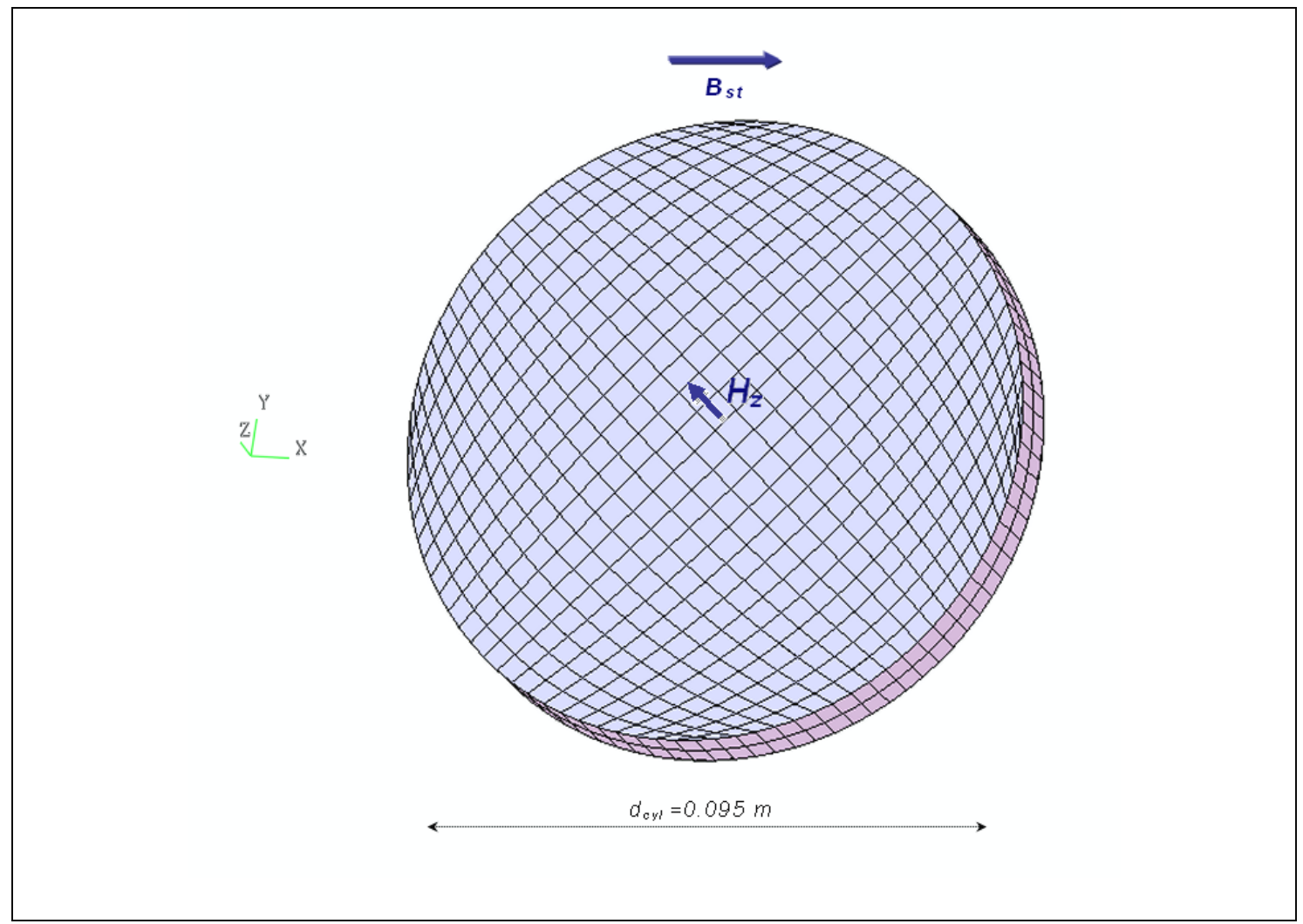

Figure 1: Geometrical model of the axially infinite plasma cylinder, with the axial component of the simulated alternating magnetic field $H_{z}$ and the superimposed static magnetic field $B_{s t}$. 
Figure 2, S. Sfikas et al

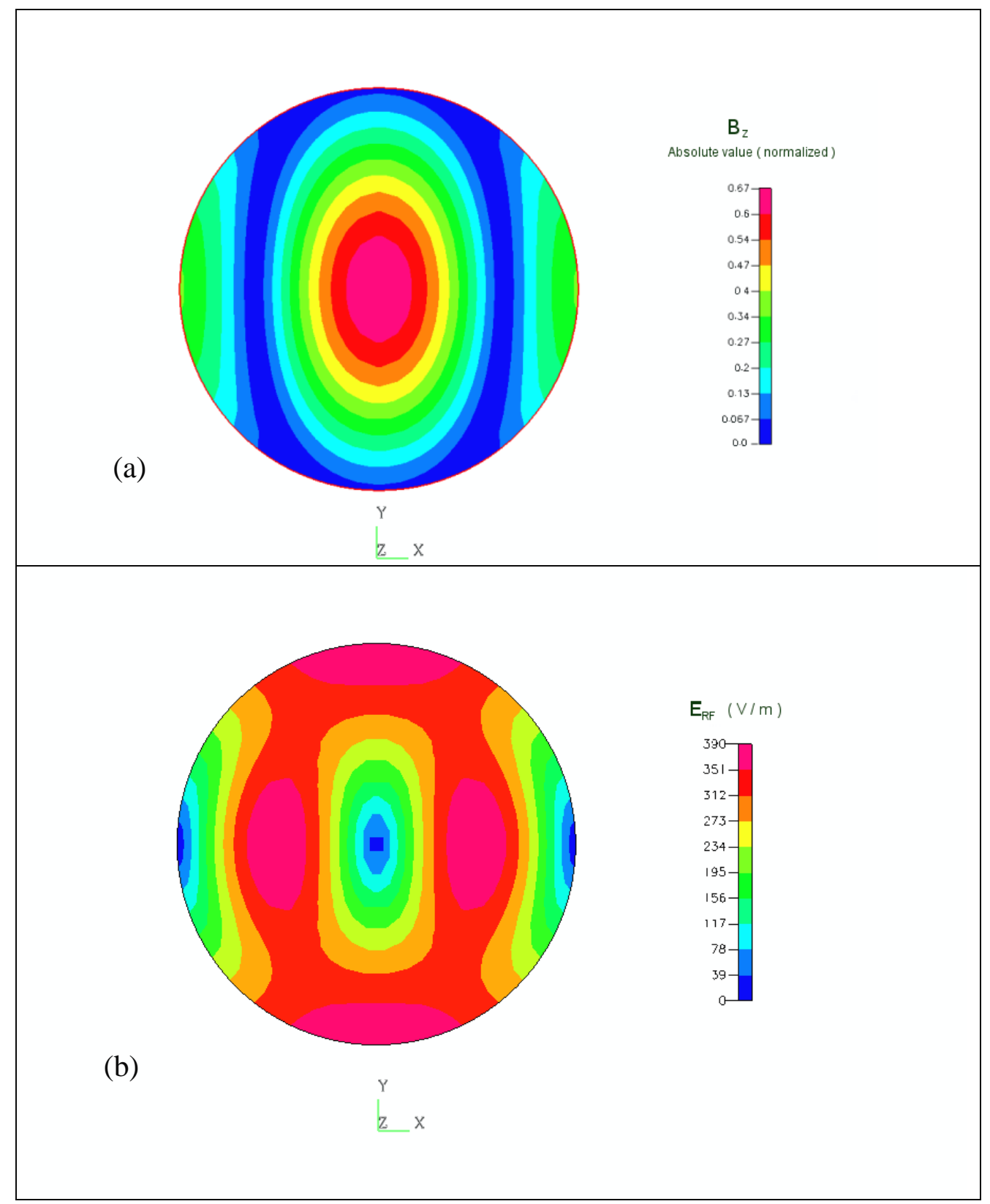

Figure 2: Maps of (a) the simulated alternating magnetic field $B_{\mathrm{Z}}$ (normalized to the field value at the walls) and (b) the magnitude of the induced electric field, resulting from the specific boundary conditions used for the magnetic potential. 
Figure 3, S. Sfikas et al

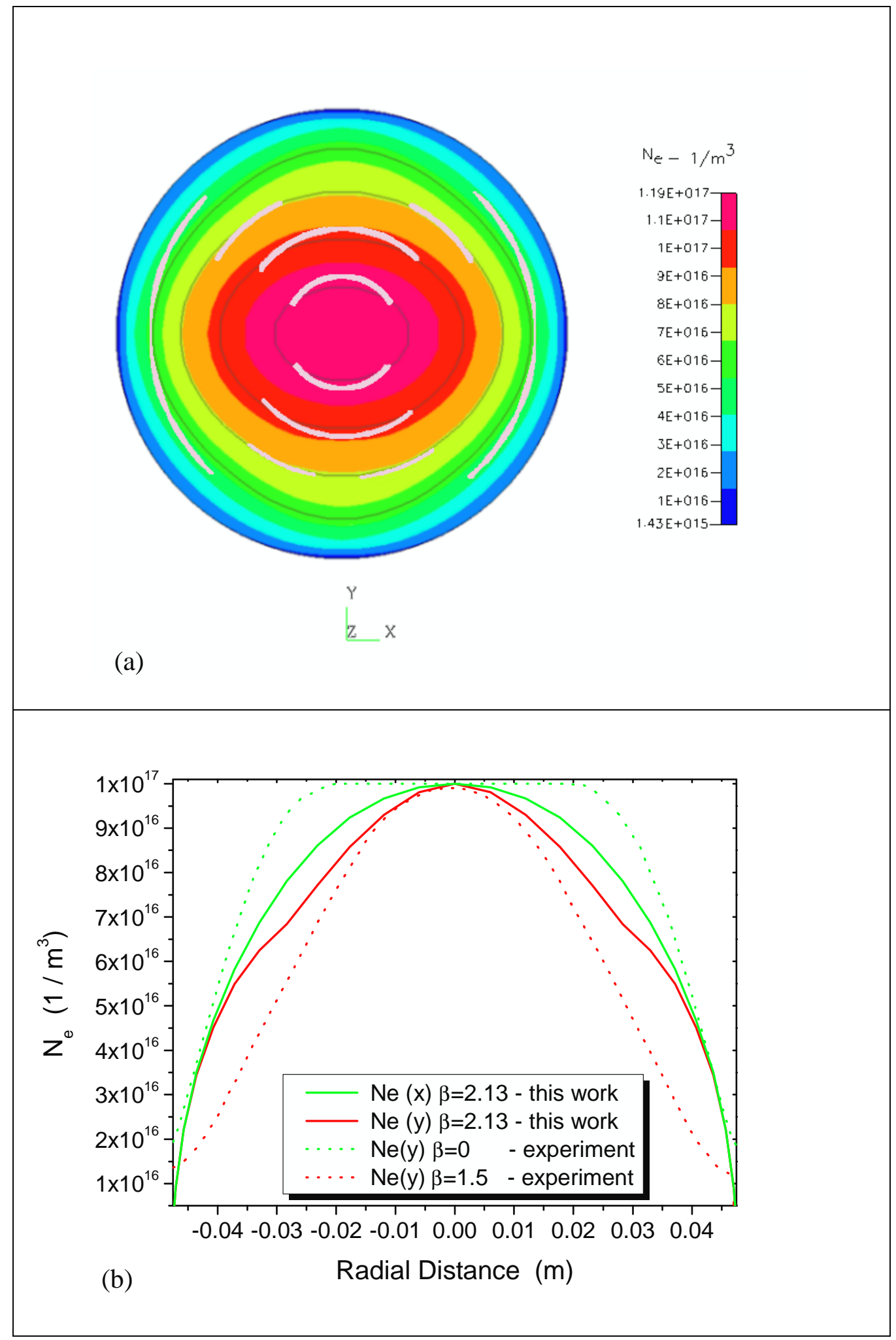

Figure 3: (a) Map of electron density $N_{\mathrm{e}}$ as calculated and compared to the experimental curves [12] of higher density regions (light colored lines) at radius $r=1$, 2, 3 and $4 \mathrm{~cm}$ and (b) Electron density profiles along $(x)$ and transverse $(y)$ direction to the static magnetic field $B_{s t}$ as calculated in the present simulation and experimentally measured in [12]. 
Figure 4, S. Sfikas et al

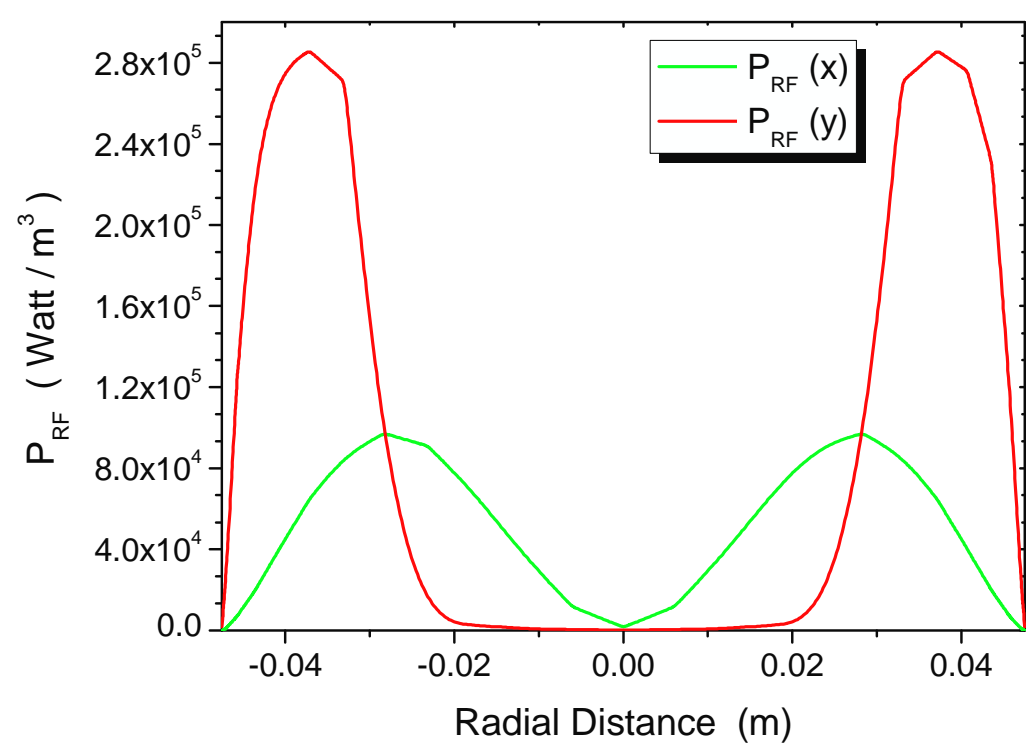

(a)

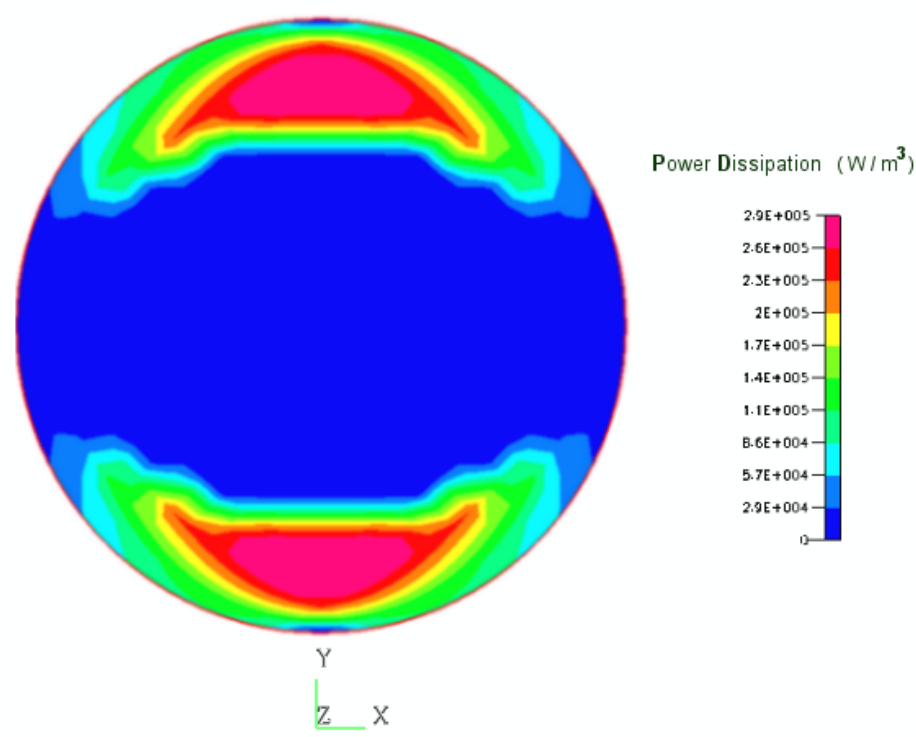

(b)

Figure 4: (a) Inductive power dissipation $P_{\mathrm{RF}}$ profile, along $(x)$ and transverse $(y)$ to the static magnetic field $B_{s t}$ (b) Inductive power dissipation $P_{\mathrm{RF}}$ spatial map. 
Figure 5, S. Sfikas et al

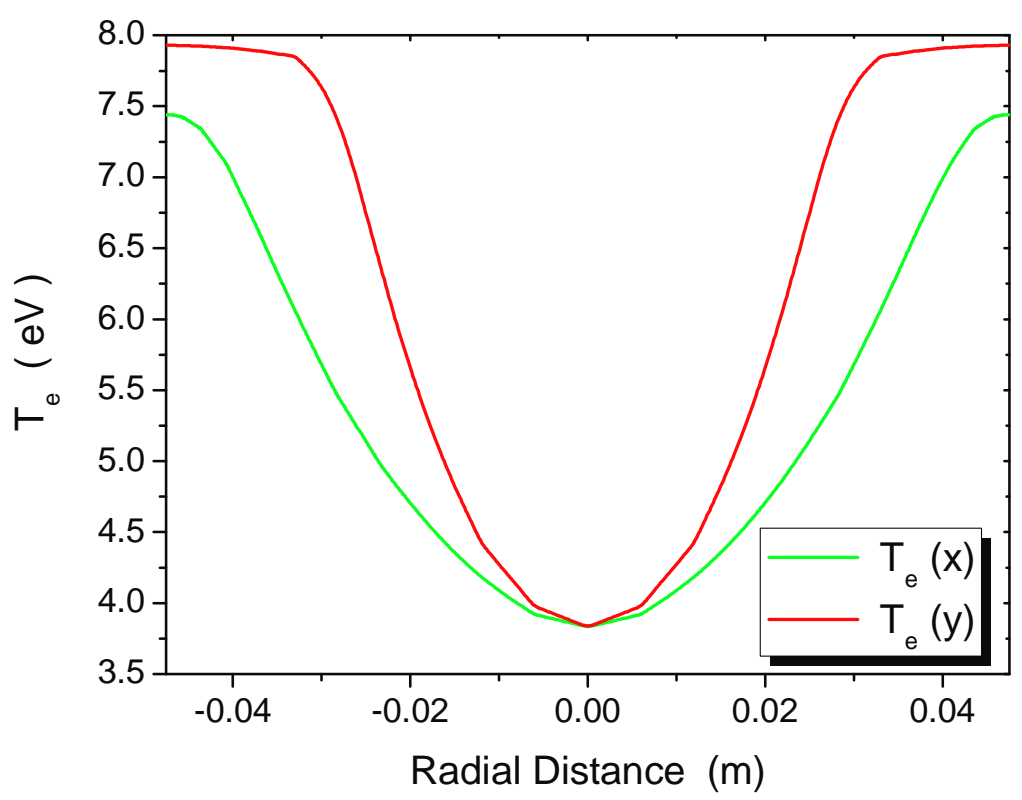

(a)

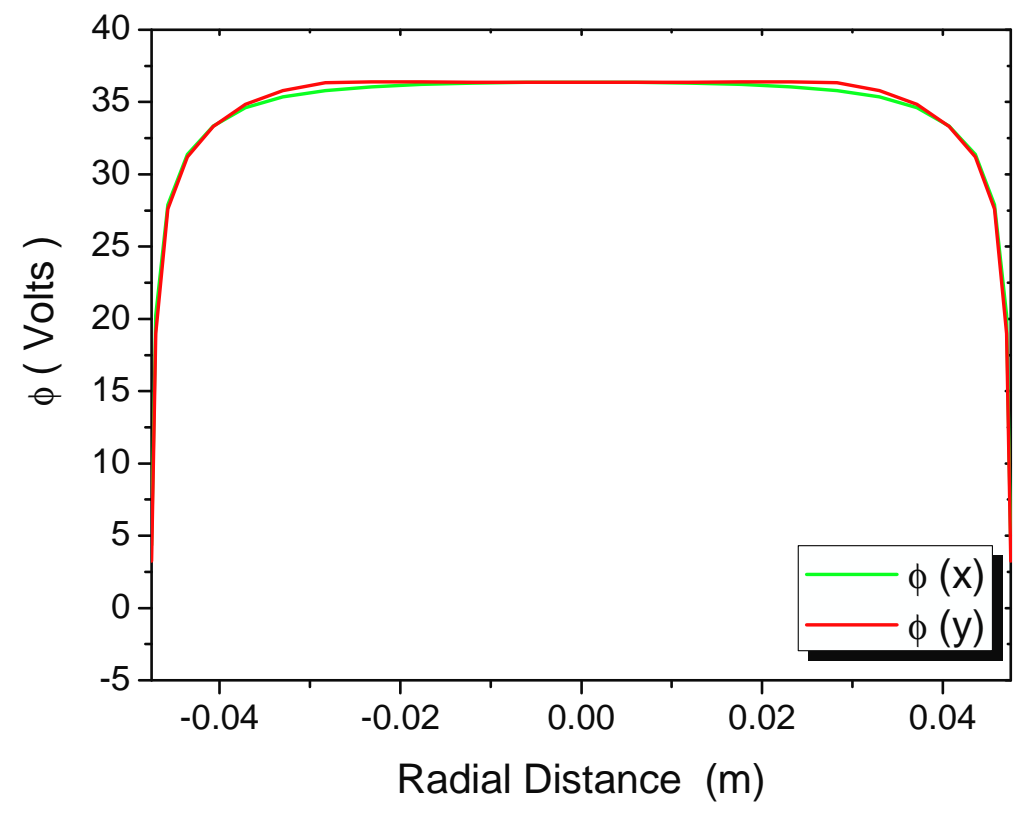

(b)

Figure 5: (a) Electron temperature $T_{\mathrm{e}}$ and (b) electric potential $\Phi$ profiles, along $(x)$ and transverse $(y)$ to the static magnetic field $B_{s t}$. 
Figure 6, S. Sfikas et al

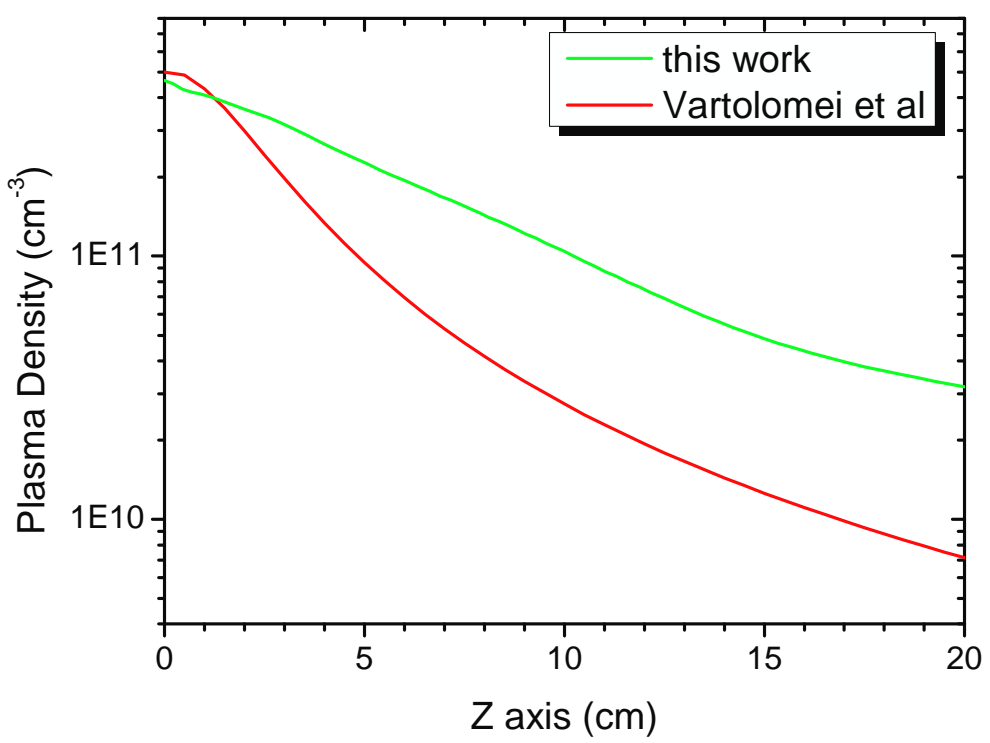

(a)

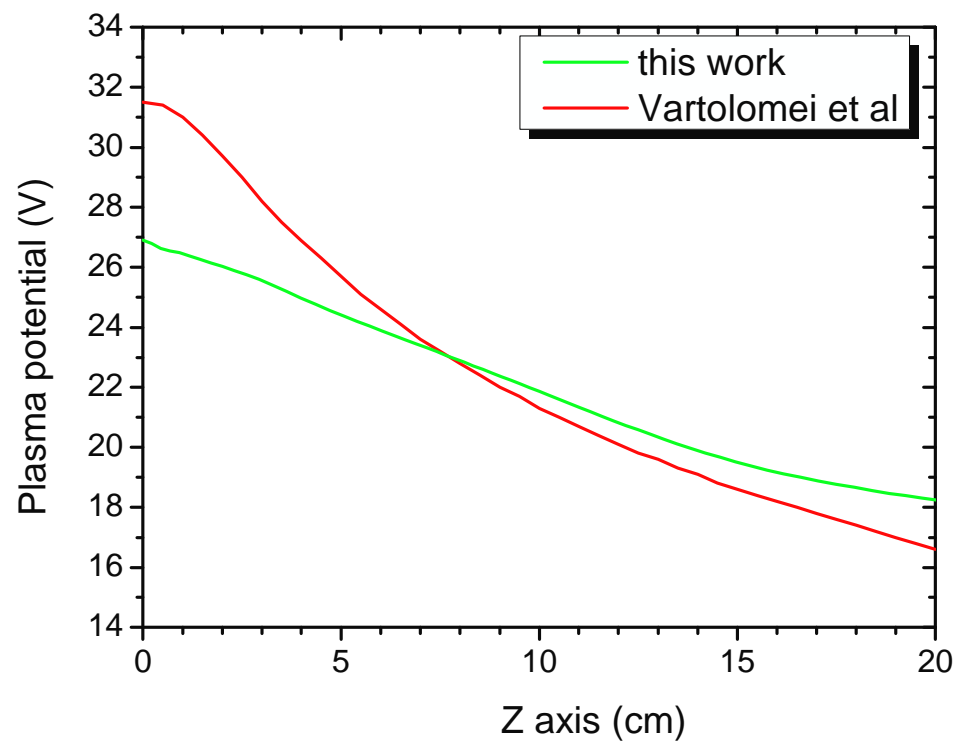

(b)

Figure 6: (a) Electron density and (b) plasma potential as a function of the distance from the center of the coil towards as calculated in the present simulation and compared to the spherical expansion model and experimental measurements of [23,24]. 